\title{
Chemistry of Polyhalogenated Nitrobutadienes, Part 11: ipso-Formylation of 2-Chlorothiophenes under Vilsmeier-Haack Conditions
}

\author{
Eva-Janina Vogt, Viktor A. Zapol'skii, Eva Nutz, and Dieter E. Kaufmann \\ Institute of Organic Chemistry, Clausthal University of Technology, Leibnizstraße 6, \\ 38678 Clausthal-Zellerfeld, Germany
}

Reprint requests to Prof. Dr. D. E. Kaufmann. Fax: +49-5323-722834.

E-mail: dieter.kaufmann@tu-clausthal.de

Z. Naturforsch. 2012, 67b, 285-294; received March 2, 2012

\begin{abstract}
The regioselective ipso-formylation of electron-rich, 3,4-push-pull-substituted 2-chlorothiophenes under Vilsmeier-Haack conditions was performed in good yields. The synthetic scope of this new reaction was explored using various halothiophenes, chloroanilines, and 1-methyl-3-chloroindole. In comparison with their structural $\mathrm{C}-\mathrm{H}$ analogs the chlorinated thiophenes, anilines, and the indole proved to be less reactive toward electrophilic attack by chloromethyleniminium salts.
\end{abstract}

Key words: Vilsmeier-Haack Formylation, Thiophene, Push-Pull Substitution, ipso-Substitution, Enamine

\section{Introduction}

Within our scope to employ 2-nitroperchlorobutadiene (1) as a versatile building block for the predictable synthesis of bioactive heterocycles [1a-d], we have also developed an efficient three-step synthesis of the 3-amino-4-nitrothiophenes 3 via the aminodithiolanes 2 [1d] (Scheme 1).

These thiophenes have a unique substitution pattern and are interesting precursors for thiophene-based optoelectronic materials [2] such as conducting polymers with small HOMO-LUMO gaps. To interconnect these thiophene monomers via $\pi$ bonds, introduction of a formyl group in 2-position of $\mathbf{3}$ was intended. The Vilsmeier reaction is known to place a formyl group regioselectively onto activated heteroaromatic ring systems such as thiophenes [3]. As a primary selective reduction of the $\mathrm{C}-\mathrm{Cl}$ group proved difficult, it was aimed to ipso-substitute this position directly. Gratifyingly, this formylation was successful. Therefore, in the present paper we have focused our efforts on the synthetic scope of the ipso-substitution of substituted 2-halothiophenes.

\section{Results and Discussion}

The Vilsmeier reaction comprises the selective electrophilic substitution of activated $\mathrm{C}-\mathrm{H}$ aromatic or heteroaromatic ring systems with $N$-derivatives of the unstable formyl chloride - chloromethyleniminium salts - being formed by reaction of $N, N$ dimethylformamide (DMF) or $N$-methylformanilide with acid chlorides such as phosphoryl chloride or phosgene [3]. So far, ipso-variants of the VilsmeierHaack formylation have been reported rarely, either with phenylmercury compounds [4] or with tert-butylcalix[4]arene [5].

Starting from 2-nitroperchlorobutadiene (1) [6], the $N$-(2-chloro-4-nitro-5-(vinylsulfanyl)-thien-3-yl)amines $3 \mathbf{a}-\mathbf{d}$ are accessible in very good yields (90$95 \%$ [1d]. Surprisingly, these persubstituted thiophene derivatives reacted in an $\mathrm{S}_{\mathrm{E}} \mathrm{Ar}$ reaction with a Vilsmeier reagent under exclusive formylation at C-2, with substitution of the chloro substituent (Scheme 2). DMF or $N$-methylformanilide were used in excess as both, reactant and solvent. Phosphoryl chloride was added at $0{ }^{\circ} \mathrm{C}$ in a small excess of $10-20 \%$, and the

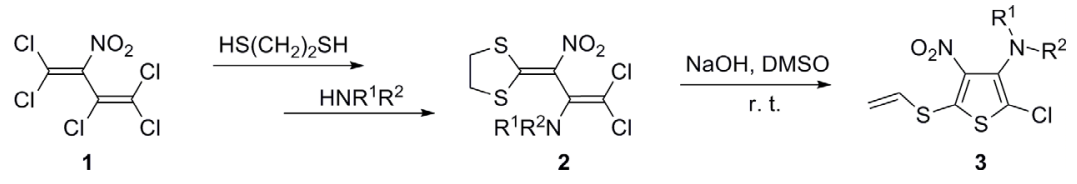

Scheme 1. Three-step synthesis of the 3-amino-4-nitrothiophenes 3. 


\begin{tabular}{cccccccc}
\hline Entry & Starting material & Solvent & $\mathrm{POCl}_{3}(\mathrm{eq})$. & $T\left({ }^{\circ} \mathrm{C}\right)$ & Reaction time $(\mathrm{h})$ & Product & Yield $(\%)$ \\
\hline 1 & $\mathbf{3 a}$ & DMF & 1.1 & 50 & 2 & $\mathbf{4 a}$ & 79 \\
2 & 3a & $\mathrm{Ph}(\mathrm{Me}) \mathrm{NCHO}$ & 1.1 & r. t. & 5 & $\mathbf{4 a}$ & 61 \\
3 & 3b & DMF & 1.2 & r. t. & 4 & $\mathbf{4 b}$ & 70 \\
4 & 3c & DMF & 1.2 & r. t. & 3 & $\mathbf{4 c}$ & 50 \\
5 & 3d & DMF & 1.2 & 50 & 5 & $\mathbf{4 d}$ & 72 \\
\hline
\end{tabular}

Table 1. Reaction conditions for the ipso-formylation of 2chlorothiophenes 3 .
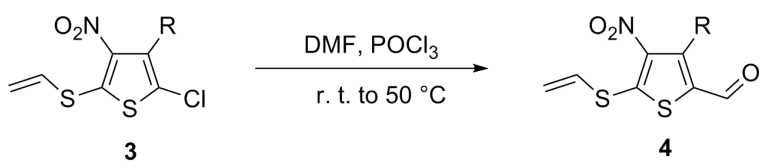

a $R=\widehat{N}^{O}(79 \%)$ c $R=-\Gamma$

$(50 \%)$

b $\mathrm{R}=\widehat{\mathrm{N}}(70 \%)$

d $\mathrm{R}=\overbrace{(72 \%)}^{\mathrm{N}^{-\mathrm{CO}_{2} \mathrm{Et}}}$

Scheme 2. Vilsmeier-Haack ipso-formylation of the persubstituted 2-chlorothiophenes 3 .

temperature was slowly raised, first to room temperature (r.t.), and then to $50{ }^{\circ} \mathrm{C}$. The resulting aldehydes $\mathbf{4 a}-\mathbf{d}$ were obtained in moderate to good yields $(50-79 \%$, Table 1$)$, without a reaction of the vinylsulfanyl group.

The substitution pattern of the tetrasubstituted thiophenes $\mathbf{3}$ is unique, comprising a combination of a chloroenamine, a push-pull system and an aminothioketenacetal, all in one leading to a very electron-rich molecule. This special electronic situation should facilitate an electrophilic attack at C-2. Although the mechanism of this new reaction is not yet fully understood [7], we became interested in its synthetic scope and limitations, the essential substitution pattern as well as the specific reaction conditions. Therefore, under the same reaction conditions we reacted aromatic haloenamines such as 2,6-dichloro- $N, N$-dimethylaniline (5) and 2,4,6-trichloro- $N, N$-dimethylaniline (6) which were synthesized according to the literature [8]. Both chloroanilines proved to be not reactive enough to get formylated, neither at $50{ }^{\circ} \mathrm{C}$ nor at $70{ }^{\circ} \mathrm{C}$ (Scheme 3).

Indoles are known to be easily attacked at C-3, even by rather weak electrophiles such as Mannich and Vilsmeier reagents. The $N$-heteroaromatic haloenamine 3-chloro-1-methyl- $1 H$-indole (8) [9], though, at $50{ }^{\circ} \mathrm{C}$ and with $1.1 \mathrm{eq}$. of phosphoryl chloride and an excess of DMF, afforded 3-chloro-1-methyl-1Hindole-2-carbaldehyde (9) as the main product with $30 \%$ yield. In accordance with the literature, C-2 is formylated in a Vilsmeier reaction, when the other-
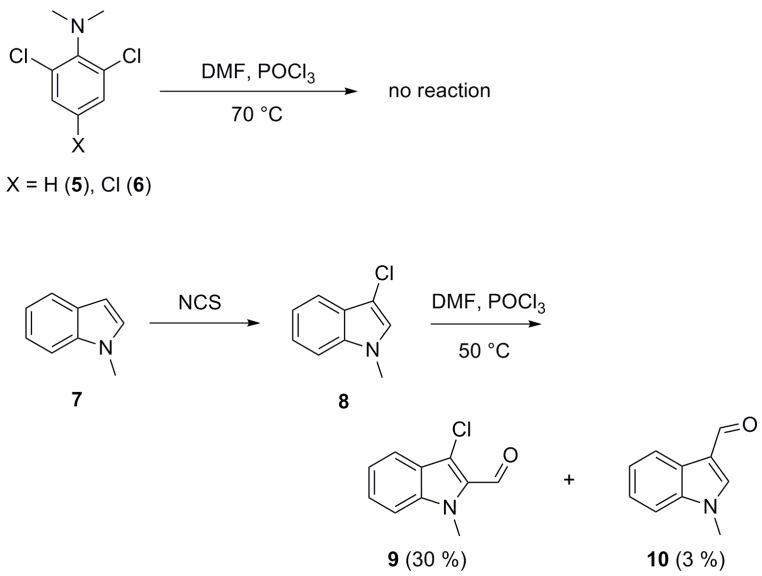

Scheme 3. Attempted ipso-formylation of chloroanilines 5 and $\mathbf{6}$ and chloroindole $\mathbf{8}$.

wise preferred C-3 position is blocked by a chlorine substituent [10]. However, 1-methyl- $1 H$-indole3-carbaldehyde (10) was found as a by-product with $3 \%$ yield showing that the ipso-Vilsmeier reaction found for the thiophenes $\mathbf{3 a}-\mathbf{d}$ is transferable to other systems (Scheme 3).

In the next step we investigated the influence of the thiophene ring and its substitution pattern on the formylation reaction of different halogenated thiophenes. Under Vilsmeier-Haack conditions with 2.2 eq. of phosphoryl chloride at $50{ }^{\circ} \mathrm{C}$ up to $100{ }^{\circ} \mathrm{C}$ the 2-halothiophenes ( $\mathrm{Cl} \mathrm{11}, \mathrm{Br} 12, \mathrm{I} 13)$ afforded the classical Vilsmeier reaction products exclusively, viz. the 5-halo-2-thiophenecarbaldehydes ( $\mathrm{Cl} \mathrm{14,} \mathrm{Br} \mathrm{15}, \mathrm{I} 16)$; with 2,5-dihalothiophenes $(\mathrm{Cl} \mathrm{17}, \mathrm{Br} 18, \mathrm{I}$ 19) under the same conditions no reaction occurred (Scheme 4).

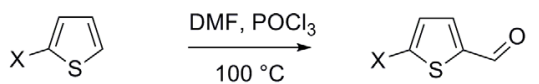

$$
\begin{aligned}
& \mathrm{X}=\mathrm{Cl} \text { (11), } \mathrm{Br}(12), \mathrm{I}(13) \quad \mathrm{X}=\mathrm{Cl}(14), \mathrm{Br}(15), \mathrm{I} \text { (16) } \\
& \underset{\mathrm{S}}{\stackrel{\mathrm{DMF}, \mathrm{POCl}_{3}}{\longrightarrow}} \underset{100^{\circ} \mathrm{C}}{\stackrel{\mathrm{C}^{2}}{\longrightarrow}} \text { no reaction } \\
& \mathrm{X}=\mathrm{Cl} \text { (17), } \mathrm{Br} \text { (18), I (19) }
\end{aligned}
$$

Scheme 4. Attempted ipso-formylation of mono- 11-13 and di-halothiophenes 17-19. 


\begin{tabular}{|c|c|c|c|c|c|c|c|}
\hline Entry & Starting material & Solvent & $\mathrm{POCl}_{3}$ (eq.) & $T\left({ }^{\circ} \mathrm{C}\right)$ & Reaction time (h) & Product & Yield (\%) \\
\hline 1 & 20 & DMF & 1.1 & 50 & 1.5 & 21 & 62 \\
\hline 2 & 22 & DMF & 1.1 & r. t., 50 & 1.5 & - & - \\
\hline 3 & 23 & DMF & 1.1 & 50 & 4.5 & 21 & 25 \\
\hline 4 & 24 & DMF & 1.1 & 50 & 1.0 & 25 & 33 \\
\hline Entry & Starting material & Solvent & $\mathrm{POCl}_{3}$ (eq.) & $T\left({ }^{\circ} \mathrm{C}\right)$ & Reaction time (h) & Product & Yield (\%) \\
\hline 1 & 26 & DMF & 1.1 & 50 & 1.5 & 27 & 83 \\
\hline 2 & 28 & DMF & 1.1 & 50 & 24 & $\begin{array}{c}\text { 30, } 31 \\
(0.32: 1)\end{array}$ & 66 \\
\hline 3 & 29 & DMF & 1.1 & 50 & 3.0 & $\begin{array}{c}\mathbf{3 0}, \mathbf{3 1} \\
(1: 0.22)\end{array}$ & 84 \\
\hline
\end{tabular}

Table 2. Reaction conditions for the ipso-formylation of 3-morpholinothiophenes $\mathbf{2 0}$ and 22-24.

Table 3. Reaction conditions for the ipso-formylation of 3,4-ethylenedioxythiophenes 26, 28 and 29.
In accordance with the literature [10-13] the halogen substituents deactivate the thiophene so that the reactions require higher temperatures $\left(100{ }^{\circ} \mathrm{C}\right)$ to take place. In both cases the halogen atoms act as protecting substituents for the 2- or 2,5-position and, additionally in case of bromine and iodine substituents, they were exchanged by chlorine to a large extent. The exchange mechanism is yet not fully understood.

Since the halothiophenes 11-13 and 17-19 did not react by ipso-substitution of the halogen while both, the 3-chloroindole $\mathbf{8}$, which reacted to the 3carbaldehyde $\mathbf{1 0}$ at least partially, as well as the aminonitrothiophenes 3 show this new reaction, apparently either an amino group or more general $+\mathrm{M}$ substituents facilitate this formylation reaction. Therefore, a number of 3-morpholinothiophenes were chosen as test compounds which were among the most stable ones in the series of $\mathbf{3 a}-\mathbf{d}$. For comparison, 4-(3-thienyl)morpholine (20), which was synthesized by a Buchwald-Hartwig reaction [15], was formylated in a Vilsmeier reaction, affording thiophene-2carbaldehyde 21 regioselectively with $62 \%$ yield. The halogenated analogs 4-(2-chloro-3-thienyl)morpholine (22) and 4-(2-bromo-3-thienyl)morpholine (23) were synthesized from 20 with NCS and NBS in DMF. 4(2,5-Dichloro-3-thienyl)morpholine (24) was synthesized from 3-bromo-2,5-dichlorothiophene according to Buchwald et al. [15]. All of these aminohalothiophenes are rather unstable, and it is not possible to store them over a long period of time. They were tested under Vilsmeier-Haack conditions with 1.1 eq. of phosphoryl chloride at r.t. to $50{ }^{\circ} \mathrm{C}$ (Scheme 5, Table 2). In the case of chlorothiophene 22 no product could be isolated but instead decomposition took place. When reacting bromothiophene $\mathbf{2 3}$, thiophene2-carbaldehyde 21 was obtained with $25 \%$ yield as the only product. Although $\mathrm{C}-5$ was not blocked by an additional substituent, the reaction occurred at $\mathrm{C}-2$
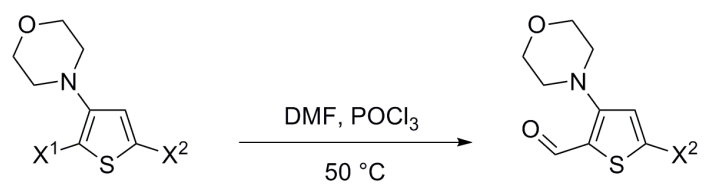

$20 \mathrm{X}^{1}=\mathrm{X}^{2}=\mathrm{H}$

$22 \mathrm{X}^{1}=\mathrm{Cl}, \mathrm{X}^{2}=\mathrm{H}$

$23 \mathrm{X}^{1}=\mathrm{Br}, \mathrm{X}^{2}=\mathrm{H}$

$24 X^{1}=X^{2}=C l$

$21 \mathrm{X}^{2}=\mathrm{H}(25-62 \%)$

$25 \mathrm{X}^{2}=\mathrm{Cl}(33 \%)$

Scheme 5. Attempted ipso-formylation of 3-morpholinothiophenes 20 and $22-24$.

next to the activating morpholino group in an ipsosubstitution of the bromo substituent. The dichlorothiophene $\mathbf{2 4}$ reacted in the same way at the activated C-2 position to give the thiophene-2-carbaldehyde $\mathbf{2 5}$ with $33 \%$ yield.

The 3-amino-4-nitro substitution of thiophenes $\mathbf{3 a}-$ d is a valuable tool for the construction of new conducting polymers. Therefore a comparison of their reactivity towards Vilsmeier reagents with the 3,4-bisalk -oxy-substituted ethylenedioxythiophene (EDOT, 26), the monomer of the most important conducting polymer polyethylenedioxythiophene (PEDOT) [16], is most interesting. The EDOT was used in classical Vilsmeier reactions and afforded as expected the 3,4ethylenedioxythiophene-2-carbaldehyde (27) in good yield $(83 \%)$. For comparison, 2,5-dibromo-3,4-ethylenedioxythiophene (28), which was synthesized according to the literature [18], and 2-bromo-3,4-ethylenedioxythiophene (29), which was synthesized under similar conditions with NBS in DMF, were reacted under Vilsmeier-Haack conditions with 1.1 eq. of phosphoryl chloride at $50{ }^{\circ} \mathrm{C}$ (Scheme 6, Table 3). Starting with $\mathbf{2 8}$ the same reaction as with the thiophenes $\mathbf{3 a}-\mathbf{d}$ took place resulting in the ipso-substitution of a bromo substituent by a formyl group. 5-Bromo-3,4-ethylenedioxythiophene-2-carbaldehyde (30) and 5-chloro-3,4ethylenedioxythiophene-2-carbaldehyde (31) were ob- 


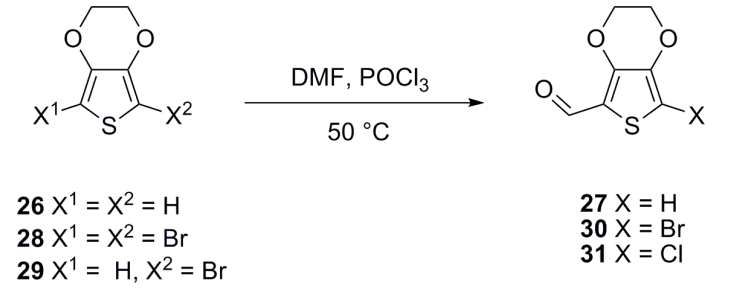

Scheme 6. ipso-Formylation of 3,4-ethylenedioxythiophenes 26, 28 and 29.

tained as a mixture $(0.32: 1)$ with $66 \%$ yield. This result shows that alkoxy substituents at C-3 also facilitate an ipso-Vilsmeier attack at position C-2. At C-5 the bromo substituent is exchanged by a chlorine atom of the phosphoryl chloride to a large extent, probably by an addition/elimination mechanism. In comparison, the reaction of $\mathbf{2 9}$ gave the same product mixture of $\mathbf{3 0}$ and 31 (1:0.22) with $84 \%$ yield. In an intramolecular competition, both $\mathrm{C}-2$ and $\mathrm{C}-5$ have the $+\mathrm{M}$ alkoxy substituent in $\alpha$-position, but the reaction only takes place at C-5 in a classical Vilsmeier reaction to give the aldehydes 30 and 31. The bromine substituent at $\mathrm{C}-2$ serves as a protecting group and is only exchanged by chlorine to a lesser extent.

\section{Conclusion}

A new and unexpected variant of the ipso-Vilsmeier reaction has been found in which a halogen is substituted by a formyl group under Vilsmeier conditions. This reaction was first developed with electron-rich thiophenes 3a-d, featuring a unique substitution pattern. In the present paper the synthetic scope of this reaction was examined. Aromatic chloroenamines such as the haloanilines $\mathbf{5}$ and $\mathbf{6}$ either did not react in this manner or only in a side reaction, like the 3-chloroindole 8. Thiophenes, though bearing an activating $+\mathrm{M}$ substituent in 3-position, show this ipso-substitution at C-2, even if C-5 remains unsubstituted such as in 4(2-bromo-3-thienyl)morpholine (23). In case of an intramolecular competition between an equally activated $\mathrm{C}-\mathrm{H}$ and $\mathrm{C}-\mathrm{Br}$ bond, formylation of the $\mathrm{C}-\mathrm{H}$ bond is preferred. So far, this new reaction is limited to $+\mathrm{M}-$ activated 2- or 2,5-halothiophenes. In the near future we will try to gain further insight into the mechanism of this reaction type.

\section{Experimental Section}

Melting points were determined with a Differential Scanning Calorimeter Perkin Elmer DSC6. Thin layer chromatography (TLC) was performed on Merck TLC-plates (alu- minum based) silica gel $60 \mathrm{~F} 254$. FT-IR spectra were obtained with a Bruker Vector 22 FT-IR spectrometer in the range of 700 to $4000 \mathrm{~cm}^{-1}$ (2.5\% pellets in $\left.\mathrm{KBr}\right)$. Mass spectra were obtained on a Varian 320 MS Triple Quad GC/MS/MS instrument with a Varian 450-GC unit usually in direct mode with electron impact $(70 \mathrm{eV})$. In the case of chlorinated and brominated compounds, all peak values of molecular ions as well as fragments refer to the isotopes ${ }^{35} \mathrm{Cl}$ and ${ }^{79} \mathrm{Br}$. The elemental composition was confirmed either by combustion analysis or by high-resolution EI and (+)ESI mass spectrometry. All HRMS results were satisfactory in comparison to the calculated accurate masses of the molecular ions ( $\pm 2 \mathrm{ppm}, \mathrm{R} \sim 10000) .{ }^{1} \mathrm{H}$ NMR $(600 \mathrm{MHz})$, ${ }^{13} \mathrm{C}$ NMR (150 MHz): Avance III $600 \mathrm{MHz}$ FT-NMR spectrometer (Bruker, Rheinstetten, Germany); ${ }^{1} \mathrm{H}$ NMR (400 MHz), ${ }^{13} \mathrm{C}$ NMR (100 MHz): Avance $400 \mathrm{FT}-\mathrm{NMR}$ spectrometer (also Bruker). ${ }^{1} \mathrm{H}$ NMR $(200 \mathrm{MHz}),{ }^{13} \mathrm{C}$ NMR (50 MHz): DPX 200 FT-NMR spectrometer (also Bruker). ${ }^{1} \mathrm{H}$ and ${ }^{13} \mathrm{C}$ NMR spectra were referenced to the residual solvent peak: $\mathrm{CDCl}_{3}, \delta=7.26\left({ }^{1} \mathrm{H}\right), \delta=77.0\left({ }^{13} \mathrm{C}\right) \mathrm{ppm}$. Chemical shifts $\delta$ are given in ppm. In most cases, peak assignments were accomplished by HSQC and HMBC NMR experiments. Purifications were carried out by means of column chromatography on silica gel 60 (Merck). Petroleum ether as eluent had the boiling range $60-70{ }^{\circ} \mathrm{C}$.

Ethyl 4-(1,1-dichloro-3-(1,3-dithiolan-2-ylidene)-3-nitro-1propen-2-yl)-1-piperazinecarboxylate (2d)

2d was synthesized as reported for the dithiolanes $\mathbf{2 a}-$ c [1d]. At r.t. ethyl 1-piperazinecarboxylate hydrochloride $(1.60 \mathrm{~g}, 10.20 \mathrm{mmol})$ was added to a solution of 2-(2,3,3trichloro-1-nitro-2-propen-1-ylidene)-1,3-dithiolane (1.00 g, $3.40 \mathrm{mmol})$ and triethylamine $(1.03 \mathrm{~g}, 10.20 \mathrm{mmol})$ in methanol $(15 \mathrm{~mL})$. The mixture was refluxed for $3 \mathrm{~d}$. After cooling to r.t. the solution was concentrated in vacuo to $40 \%$ of its volume. Water $(5 \mathrm{~mL})$ was added, and the resulting solid was filtered, washed with water $(3 \times 10 \mathrm{~mL})$ and dried in vacuo. The product was isolated as a red solid; yield: 1.33 g (95\%), m. p. $59{ }^{\circ} \mathrm{C} .-{ }^{1} \mathrm{H}$ NMR (200 MHz, $\left.\mathrm{CDCl}_{3}\right)$ : $\delta=1.23\left(\mathrm{t}, J=7.1 \mathrm{~Hz}, 3 \mathrm{H}, \mathrm{CH}_{3}\right), 2.98\left(\mathrm{bs}, 4 \mathrm{H}, \mathrm{CH}_{2} \mathrm{NCH}_{2}\right)$, $3.46-3.64\left(\mathrm{~m}, 8 \mathrm{H}, \mathrm{CH}_{2} \mathrm{NCH}_{2}+\mathrm{S}\left(\mathrm{CH}_{2}\right)_{2} \mathrm{~S}\right), 4.13$ (q, $J=$ $\left.7.1 \mathrm{~Hz}, 2 \mathrm{H}, \mathrm{CH}_{2}\right) .-{ }^{13} \mathrm{C} \mathrm{NMR}\left(50 \mathrm{MHz}, \mathrm{CDCl}_{3}\right): \delta=14.6$ $\left(1 \mathrm{C}, \mathrm{CH}_{3}\right), 37.6\left(1 \mathrm{C}, \mathrm{SCH}_{2}\right), 40.1\left(1 \mathrm{C}, \mathrm{SCH}_{2}\right), 43.8$ (1 C, $\left.\mathrm{CH}_{2}\right), 49.1$ ( $\left.2 \mathrm{C}, \mathrm{CH}_{2} \mathrm{NCH}_{2}\right), 61.4\left(2 \mathrm{C}, \mathrm{CH}_{2} \mathrm{NCH}_{2}\right), 114.1$ $\left(1 \mathrm{C}, C \mathrm{Cl}_{2}\right), 130.5\left(1 \mathrm{C}, C-\mathrm{NO}_{2}\right), 139.0\left(1 \mathrm{C}, C-\left(\mathrm{N}\left(\mathrm{CH}_{2}\right)_{2}\right)\right)$, 155.3 ( $1 \mathrm{C}, \mathrm{COO}), 170.7$ (1 C, SCS). - IR (KBr): $v=2980$, 2906, 2845, 2605, 2498, 1696, 1584, 1522, 1461, 1431, 1383, 1346, 1295, 1276, 1248, 1136, 1118, 1074, 1022, 989, 949, 912, 850, 799, 775, $711 \mathrm{~cm}^{-1}$. - MS (EI, $\left.70 \mathrm{eV}\right)$ : $m / z(\%)=414(27)[\mathrm{M}+\mathrm{H}]^{+}, 350(37), 285(49), 210(77)$, 130 (88), 105 (46), 70 (64), 56 (100). - HRMS ((+)-ESI): $m / z=414.0114$ (calcd. 414.0116 for $\mathrm{C}_{13} \mathrm{H}_{18} \mathrm{Cl}_{2} \mathrm{~N}_{3} \mathrm{O}_{4} \mathrm{~S}_{2}$, $\left.[\mathrm{M}+\mathrm{H}]^{+}\right)$. 
Ethyl 4-(2-chloro-4-nitro-5-(vinylsulfanyl)-3-thienyl)-1piperazinecarboxylate $(3 \mathrm{~d})$

3d was synthesized according to the literature [1d]. An aqueous (aq.) solution of $\mathrm{NaOH}(40 \%, 1.00 \mathrm{~g}, 10.0 \mathrm{mmol})$ was added to a solution of ethyl 4-(1,1-dichloro-3-(1,3dithiolan-2-ylidene)-3-nitro-1-propen-2-yl)-1 -piperazinecarboxylate (2d) $(826 \mathrm{mg}, 2.00 \mathrm{mmol})$ in DMSO $(10 \mathrm{~mL})$ at $0{ }^{\circ} \mathrm{C}$ within $10 \mathrm{~min}$. After $1 \mathrm{~h}$ at $0{ }^{\circ} \mathrm{C}$ the mixture was stirred for an additional $3 \mathrm{~h}$ at r.t. Then cold water $(70 \mathrm{~mL})$ was added at 0 to $5{ }^{\circ} \mathrm{C}$. The mixture was acidified to $\mathrm{pH}=1$ upon dropwise addition of conc. aq. $\mathrm{HCl}$. The product precipitated as an oil, and the aq. fraction was decanted. The oily product was dissolved in chloroform $(50 \mathrm{~mL})$, the solution was washed with aq. $\mathrm{HCl}(15 \%, 3 \times 30 \mathrm{~mL})$ and water $(2 \times 30 \mathrm{~mL})$, and was then dried (sodium sulfate). The solvent was evaporated and the product isolated as an orange solid; yield: $679 \mathrm{mg}(90 \%)$, m.p. $109{ }^{\circ} \mathrm{C} .-{ }^{1} \mathrm{H}$ NMR $\left(200 \mathrm{MHz}, \mathrm{CDCl}_{3}\right): \delta=1.26\left(\mathrm{t}, J=7.1 \mathrm{~Hz}, 3 \mathrm{H}, \mathrm{CH}_{3}\right), 3.11-$ $3.17\left(\mathrm{~m}, 4 \mathrm{H}, \mathrm{CH}_{2} \mathrm{NCH}_{2}\right), 3.54-3.59\left(\mathrm{~m}, 4 \mathrm{H}, \mathrm{CH}_{2} \mathrm{NCH}_{2}\right)$, $4.14\left(\mathrm{q}, J=7.1 \mathrm{~Hz}, 2 \mathrm{H}, \mathrm{CH}_{2}\right), 5.82(\mathrm{~d}, J=9.2 \mathrm{~Hz}, 1 \mathrm{H}$, $\left.\mathrm{SCHCH}_{2, c i s}\right), 5.87\left(\mathrm{~d}, J=16.4 \mathrm{~Hz}, 1 \mathrm{H}, \mathrm{SCHCH}_{2, \text { trans }}\right), 6.52$ $(\mathrm{dd}, J=16.4,9.2 \mathrm{~Hz}, 1 \mathrm{H}, \mathrm{SCH}) .-{ }^{13} \mathrm{C} \mathrm{NMR}(50 \mathrm{MHz}$, $\left.\mathrm{CDCl}_{3}\right): \delta=14.6\left(1 \mathrm{C}, \mathrm{CH}_{3}\right), 44.4\left(2 \mathrm{C}, \mathrm{CH}_{2} \mathrm{NCH}_{2}\right), 49.9$ $\left(2 \mathrm{C}, \mathrm{CH}_{2} \mathrm{NCH}_{2}\right), 61.4\left(1 \mathrm{C}, \mathrm{CH}_{2}\right), 119.5(1 \mathrm{C}, \mathrm{C}-\mathrm{Cl}), 126.4$ $\left(1 \mathrm{C}, \mathrm{SCHCH}_{2}\right), 126.6(1 \mathrm{C}, \mathrm{SCH}), 139.2\left(1 \mathrm{C}, C-\mathrm{NO}_{2}\right)$, $140.9\left(1 \mathrm{C}, C-\left(\mathrm{N}\left(\mathrm{CH}_{2}\right)_{2}\right)\right), 141.2(1 \mathrm{C}, C-\mathrm{SCH}) 155.5(1 \mathrm{C}$, COO). - IR (KBr): $v=2977,2959,2899,2850,1699,1590$, $1546,1484,1471,1426,1384,1357,1327,1278,1244$, 1222, 1172, 1119, 1082, 1053, 1032, 994, 972, 933, 874, $830,813,767,719 \mathrm{~cm}^{-1}$. - MS (EI, $\left.70 \mathrm{eV}\right): m / z(\%)=376$ (16) $[\mathrm{M}]^{+}, 258$ (10), 233 (16), 217 (18), 195 (17), 181 (14), 144 (24), 130 (13), 116 (40), 103 (18), 83 (91), 72 (44), 56 (100). $-\mathrm{C}_{13} \mathrm{H}_{16} \mathrm{ClN}_{3} \mathrm{O}_{4} \mathrm{~S}_{2}$ (377.87): calcd. C 41.32, $\mathrm{H} 4.27$, $\mathrm{N}$ 11.12, S 16.97, Cl 9.38; found C 41.63, H 4.23, N 11.10, $\mathrm{S} 17.05, \mathrm{Cl} 9.40$.

\section{General procedure for all formylation reactions under Vilsmeier-Haack conditions}

To a solution of the starting material in anhydrous DMF or dimethylformanilide under an atmosphere of nitrogen and at $0{ }^{\circ} \mathrm{C}, 1.1-2.2$ eq. of phosphoryl chloride $\left(\mathrm{POCl}_{3}\right)$ was added slowly by a syringe. The solution was then heated to a temperature between r.t. and $100{ }^{\circ} \mathrm{C}$ for $1-24 \mathrm{~h}$ (TLC control) under stirring. After cooling to r.t. the reaction mixture was poured into ice water and stirred for $10 \mathrm{~min}$. In case of the morpholino derivative 4a in DMF the precipitate was filtered, washed with water $(3 \times 20 \mathrm{~mL})$, dried in vacuo and purified by column chromatography. In general, for the aminonitrothiophenes $\mathbf{4 a}-\mathbf{d}$ the aq. fraction was extracted with ethyl acetate $(3 \times)$, and the organic fraction was dried (magnesium sulfate). After evaporation of the solvent the product was purified by column chromatography. In all other cases a sat. aq. solution of sodium hydrogen carbonate was added. The mixture was stirred for $1 \mathrm{~h}$ and extracted with dichloromethane $(3 \times)$. The organic fraction was dried (magnesium sulfate). After evaporation of the solvent the products were purified by column chromatography.

\section{3-(4-Morpholino)-4-nitro-5-(vinylsulfanyl)thiophene-2- carbaldehyde (4a)}

Method I: The product was synthesized according to the general procedure from 4-(2-chloro-4-nitro-5-(vinylsulfanyl)-3-thienyl)morpholine (3a) (2.50 g, $8.2 \mathrm{mmol}$ ), anhydrous DMF (15 mL), and 1.1 eq. of $\mathrm{POCl}_{3}(0.82 \mathrm{~mL}, 9.0 \mathrm{mmol})$; $50{ }^{\circ} \mathrm{C}, 2 \mathrm{~h}$, ice water $(110 \mathrm{~mL})$; column chromatography (petroleum ether-ethyl acetate $3: 1$ ). The product was isolated as an orange solid; yield: $1.944 \mathrm{~g}(79 \%)$.

Method II: The product was synthesized according to the general procedure from 4-(2-chloro-4-nitro-5-(vinylsulfanyl)-3-thienyl)morpholine (3a) (100 mg, $0.327 \mathrm{mmol}$ ), dimethylformanilide $(1 \mathrm{~mL})$, and 1.1 eq. of $\mathrm{POCl}_{3}$ (0.033 mL, $0.360 \mathrm{mmol})$; r.t., $5 \mathrm{~h}$, ice water $(5 \mathrm{~mL})$, ethyl acetate $(3 \times 15 \mathrm{~mL})$; column chromatography (petroleum ether-ethyl acetate $4: 1$ ). The product was isolated as an orange solid; yield $59 \mathrm{mg}(61 \%)$, m.p. $155{ }^{\circ} \mathrm{C} .-{ }^{1} \mathrm{H}$ NMR (400 MHz, $\left.\mathrm{CDCl}_{3}\right): \delta=3.39-3.41\left(\mathrm{~m}, 4 \mathrm{H}, \mathrm{CH}_{2} \mathrm{NCH}_{2}\right)$, $3.88-3.90\left(\mathrm{~m}, 4 \mathrm{H}, \mathrm{CH}_{2} \mathrm{OCH}_{2}\right), 5.99(\mathrm{~d}, J=9.2 \mathrm{~Hz}, 1 \mathrm{H}$, $\left.\mathrm{SCHCH}_{2, c i s}\right), 6.02\left(\mathrm{~d}, J=16.4 \mathrm{~Hz}, 1 \mathrm{H}, \mathrm{SCHCH}_{2, \text { trans }}\right.$ ), $6.65(\mathrm{dd}, J=16.4,9.2 \mathrm{~Hz}, 1 \mathrm{H}, \mathrm{SCH}), 10.01(\mathrm{~s}, 1 \mathrm{H}$, $\mathrm{CHO}) .-{ }^{13} \mathrm{C}$ NMR $\left(100 \mathrm{MHz}, \mathrm{CDCl}_{3}\right): \delta=53.6(2 \mathrm{C}$, $\mathrm{CH}_{2} \mathrm{NCH}_{2}$ ), 66.7 (2 C, $\mathrm{CH}_{2} \mathrm{OCH}_{2}$ ), 124.5 (1 C, $C-\mathrm{CHO}$ ), $125.4(1 \mathrm{C}, \mathrm{SCH}), 128.7\left(1 \mathrm{C}, \mathrm{SCHCH}_{2}\right), 136.0$ (1 C, $C$ $\left.\mathrm{NO}_{2}\right), 150.3\left(1 \mathrm{C}, C-\left(\mathrm{N}\left(\mathrm{CH}_{2}\right)_{2}\right)\right), 159.8$ (1 C, $\left.C-\mathrm{SCH}\right), 180.1$ (1 C, CHO). - IR (KBr): $v=2972,2872,1624,1533,1490$, $1446,1424,1376,1352,1332,1304,1264,1223,1137$, 1111, 1067, 1021, 996, 965, 951, 867, 848, 824, 783, 758, $713 \mathrm{~cm}^{-1}$. - MS (EI, $\left.70 \mathrm{eV}\right): m / z(\%)=301(3)[\mathrm{M}]^{+}, 283$ (6) $[\mathrm{M}-\mathrm{O}]^{+}, 265$ (14), 237 (14), 227 (19), 211 (13), 197 (10), 183 (11), 168 (15), 155 (19), 137 (17), 123 (18), 112 (38), 95 (47), 73 (100), 84 (35), 69 (100), 59 (56). - HRMS (EI): $m / z=300.0235$ (calcd. 300.0239 for $\mathrm{C}_{11} \mathrm{H}_{12} \mathrm{~N}_{2} \mathrm{O}_{4} \mathrm{~S}_{2}$, $\left.[\mathrm{M}]^{+}\right)$.

4-Nitro-3-(1-piperidinyl)-5-(vinylsulfanyl)thiophene-2-carbaldehyde $(\mathbf{4 b})$

The product was synthesized according to the general procedure from 4-(2-chloro-4-nitro-5-(vinylsulfanyl)-3-thienyl)piperidine (3b) $(608 \mathrm{mg}, 2.0 \mathrm{mmol})$, anhydrous DMF $(4 \mathrm{~mL})$, and 1.2 eq. of $\mathrm{POCl}_{3}(0.22 \mathrm{~mL}, 2.4 \mathrm{mmol})$; r.t., $4 \mathrm{~h}$, ice water $(5 \mathrm{~mL})$, ethyl acetate $(3 \times 30 \mathrm{~mL})$; column chromatography (petroleum ether-ethyl acetate $1: 1$ ). The product was isolated as a red solid; yield: $417 \mathrm{mg}(70 \%)$, m.p. $133{ }^{\circ} \mathrm{C} .-{ }^{1} \mathrm{H}$ NMR $\left(200 \mathrm{MHz}, \mathrm{CDCl}_{3}\right): \delta=1.60-1.83$ $\left(\mathrm{m}, 6 \mathrm{H},\left(\mathrm{CH}_{2}\right)_{3}\right), 3.30-3.35\left(\mathrm{~m}, 4 \mathrm{H}, \mathrm{CH}_{2} \mathrm{NCH}_{2}\right), 5.94$ (d, 
$\left.J=9.2 \mathrm{~Hz}, 1 \mathrm{H}, \mathrm{SCHCH}_{2, c i s}\right), 5.98(\mathrm{~d}, J=16.4 \mathrm{~Hz}, 1 \mathrm{H}$, $\left.\mathrm{SCHCH}_{2, \text { trans }}\right), 6.65$ (dd, $\left.J=16.4,9.2 \mathrm{~Hz}, 1 \mathrm{H}, \mathrm{SCH}\right), 9.97$ (s, $1 \mathrm{H}, \mathrm{CHO}) .-{ }^{13} \mathrm{C}$ NMR $\left(50 \mathrm{MHz}, \mathrm{CDCl}_{3}\right): \delta=23.6(1 \mathrm{C}$, $\mathrm{CH}_{2}$ ), 25.9 (2 C, $\mathrm{CH}_{2}$ ), $55.1\left(2 \mathrm{C}, \mathrm{CH}_{2} \mathrm{NCH}_{2}\right), 122.9$ (1 C, $C-\mathrm{CHO}), 125.7(1 \mathrm{C}, \mathrm{SCH}), 128.2\left(1 \mathrm{C}, \mathrm{SCHCH}_{2}\right), 136.0$ $\left(1 \mathrm{C}, C-\mathrm{NO}_{2}\right), 151.9\left(1 \mathrm{C}, C-\left(\mathrm{N}\left(\mathrm{CH}_{2}\right)_{2}\right)\right), 159.1(1 \mathrm{C}, C$ $\mathrm{SCH}), 180.1$ (1 C, CHO). - IR (KBr): $v=3088,3002,2952$, 2939, 2921, 2846, 1617, 1531, 1486, 1462, 1443, 1423, 1374, 1352, 1342, 1309, 1277, 1228, 1159, 1149, 1103, $1045,995,966,942,915,859,813,776,760,713 \mathrm{~cm}^{-1}$. MS (EI, $70 \mathrm{eV}): m / z(\%)=299(38)[\mathrm{M}+\mathrm{H}]^{+}, 281(44), 263$ (100), 250 (24), 237 (62), 224 (23), 209 (40), 199 (28), 183 (25), 164 (29), 150 (21), 136 (22), 123 (20), 112 (32), 96 (39), 84 (42), 69 (68), 55 (64). - HRMS (EI): $m / z=298.0500$ (calcd. 298.0446 for $\mathrm{C}_{12} \mathrm{H}_{14} \mathrm{~N}_{2} \mathrm{O}_{3} \mathrm{~S}_{2},[\mathrm{M}]^{+}$).

\section{4-Nitro-3-(1-pyrrolidinyl)-5-(vinylsulfanyl)thiophene-2- carbaldehyde $(4 \mathrm{c})$}

The product was synthesized according to the general procedure from 4-(2-chloro-4-nitro-5-(vinylsulfanyl)-3-thienyl)pyrrolidine (3c) $(580 \mathrm{mg}, 2.0 \mathrm{mmol})$, anhydrous DMF (4 mL), and 1.2 eq. of $\mathrm{POCl}_{3}(0.22 \mathrm{~mL}, 2.4 \mathrm{mmol})$; r. t., $4 \mathrm{~h}$, ice water $(5 \mathrm{~mL})$, ethyl acetate $(3 \times 30 \mathrm{~mL})$; column chromatography (petroleum ether-ethyl acetate $1: 1$ ). The product was isolated as a red-brown solid; yield: $284 \mathrm{mg}(50 \%)$, m. p. $161{ }^{\circ} \mathrm{C} .-{ }^{1} \mathrm{H}$ NMR $\left(200 \mathrm{MHz}, \mathrm{CDCl}_{3}\right): \delta=2.04-2.10$ $\left(\mathrm{m}, 6 \mathrm{H},\left(\mathrm{CH}_{2}\right)_{2}\right), 3.40-3.47\left(\mathrm{~m}, 4 \mathrm{H}, \mathrm{CH}_{2} \mathrm{NCH}_{2}\right), 5.91(\mathrm{~d}$, $\left.J=9.2 \mathrm{~Hz}, 1 \mathrm{H}, \mathrm{SCHCH}_{2, c i s}\right), 5.95(\mathrm{~d}, J=16.4 \mathrm{~Hz}, 1 \mathrm{H}$, $\left.\mathrm{SCHCH}_{2, \text { trans }}\right), 6.65(\mathrm{dd}, J=16.4,9.2 \mathrm{~Hz}, 1 \mathrm{H}, \mathrm{SCH}), 9.72$ (s, $1 \mathrm{H}, \mathrm{CHO}) .-{ }^{13} \mathrm{C}$ NMR $\left(50 \mathrm{MHz}, \mathrm{CDCl}_{3}\right): \delta=26.0$ $\left(2 \mathrm{C},\left(\mathrm{CH}_{2}\right)_{2}\right), 54.8\left(2 \mathrm{C}, \mathrm{CH}_{2} \mathrm{NCH}_{2}\right), 117.5(1 \mathrm{C}, \mathrm{C}-\mathrm{CHO})$, $125.3(1 \mathrm{C}, \mathrm{SCH}), 127.8\left(1 \mathrm{C}, \mathrm{SCHCH}_{2}\right), 145.5(1 \mathrm{C}, C-$ $\left.\mathrm{NO}_{2}\right), 157.3(1 \mathrm{C}, C-\mathrm{SCH}), 162.7\left(1 \mathrm{C}, C-\left(\mathrm{N}\left(\mathrm{CH}_{2}\right)_{2}\right)\right), 180.1$ (1 C, CHO). - IR (KBr): $v=3088,3033,2991,2956,2882$, 2842, 1597, 1539, 1498, 1453, 1432, 1371, 1351, 1330, 1310, 1276, 1247, 1234, 1176, 1132, 1110, 1045, 1019, 966, 942, 874, 834, 795, 757, 741, $711 \mathrm{~cm}^{-1}$. - MS (EI, $\left.70 \mathrm{eV}\right)$ : $m / z(\%)=285(3)[\mathrm{M}+\mathrm{H}]^{+}, 267(16), 249(22), 211(12)$, 196 (10), 184 (20), 151 (24), 140 (18), 111 (27), 97 (48), 84 (45), 70 (100), 59 (41). - HRMS (EI): $m / z=284.0288$ (calcd. 284.0289 for $\mathrm{C}_{11} \mathrm{H}_{12} \mathrm{~N}_{2} \mathrm{O}_{3} \mathrm{~S}_{2},[\mathrm{M}]^{+}$).

\section{Ethyl 4-(2-formyl-4-nitro-5-(vinylsulfanyl)-3-thienyl)-1- piperazinecarboxylate $(\mathbf{4 d})$}

The product was synthesized according to the general procedure from $754 \mathrm{mg}$ (2.0 mmol) ethyl 4-(2-chloro-4-nitro-5(vinylsulfanyl)-3-thienyl)-1-piperazinecarboxylate (3d), anhydrous DMF $(4 \mathrm{~mL})$, and 1.2 eq. of $\mathrm{POCl}_{3}(0.22 \mathrm{~mL}$, $2.4 \mathrm{mmol}) ; 50{ }^{\circ} \mathrm{C}, 5 \mathrm{~h}$, ice water $(5 \mathrm{~mL})$, ethyl acetate $(3 \times 30 \mathrm{~mL})$; column chromatography (petroleum ether-ethyl acetate $1: 1)$. The product was isolated as an orange solid; yield: $534 \mathrm{mg}(72 \%)$, m.p. $138{ }^{\circ} \mathrm{C} .-{ }^{1} \mathrm{H}$ NMR (600 MHz,
$\mathrm{CDCl}_{3}$ ): $\delta=1.28$ (t, $J=7.1 \mathrm{~Hz}, 3 \mathrm{H}, \mathrm{CH}_{3}$ ), 3.33 (bs, $4 \mathrm{H}$, $\mathrm{CH}_{2} \mathrm{NCH}_{2}$ ), $3.67-3.68\left(\mathrm{~m}, 4 \mathrm{H}, \mathrm{CH}_{2} \mathrm{NCH}_{2}\right), 4.17$ (q, $J=$ $\left.7.1 \mathrm{~Hz}, 2 \mathrm{H}, \mathrm{CH}_{2}\right), 5.97\left(\mathrm{~d}, J=9.1 \mathrm{~Hz}, 1 \mathrm{H}, \mathrm{SCHCH}_{2, c i s}\right)$, $6.00\left(\mathrm{~d}, J=16.4 \mathrm{~Hz}, 1 \mathrm{H}, \mathrm{SCHCH}_{2, \text { trans }}\right), 6.63$ (dd, $J=$ 16.4, 9.1 Hz, 1 H, SCH), 9.96 (s, $1 \mathrm{H}, \mathrm{CHO}) .-{ }^{13} \mathrm{C} \mathrm{NMR}$ $\left(150 \mathrm{MHz}, \mathrm{CDCl}_{3}\right): \delta=14.6\left(1 \mathrm{C}, \mathrm{CH}_{3}\right), 43.8(2 \mathrm{C}$, $\mathrm{CH}_{2} \mathrm{NCH}_{2}$ ), $53.2\left(2 \mathrm{C}, \mathrm{CH}_{2} \mathrm{NCH}_{2}\right), 61.7\left(1 \mathrm{C}, \mathrm{CH}_{2}\right), 125.6$ $(1 \mathrm{C}, \mathrm{SCH}), 125.6(1 \mathrm{C}, \mathrm{C}-\mathrm{CHO}), 128.8\left(1 \mathrm{C}, \mathrm{SCHCH}_{2}\right)$, $136.4\left(1 \mathrm{C}, C-\mathrm{NO}_{2}\right), 150.5\left(1 \mathrm{C}, C-\left(\mathrm{N}\left(\mathrm{CH}_{2}\right)_{2}\right)\right), 155.4(1 \mathrm{C}$, COO), 159.6 (1 C, $C$-SCH), 180.1 ( $1 \mathrm{C}, C H O)$. - IR (KBr): $v=2981,2922,2850,1703,1619,1534,1486,1467,1455$, $1436,1424,1376,1352,1311,1285,1244,1222,1135$, 1120, 1076, 1048, 1035, 999, 969, 945, $764 \mathrm{~cm}^{-1}$. - MS (EI, $70 \mathrm{eV}): m / z(\%)=371(2.5)[\mathrm{M}]^{+}, 354(11), 336(15)$, 227 (100), 210 (29), 197 (19), 183 (21), 168 (17), 155 (23), 144 (32), 130 (17), 116 (30), 97 (19). - HRMS ((+)-ESI): $m / z=394.0505$ (calcd. 394.0507 for $\mathrm{C}_{14} \mathrm{H}_{17} \mathrm{~N}_{3} \mathrm{O}_{5} \mathrm{~S}_{2} \mathrm{Na}$, $\left.[\mathrm{M}+\mathrm{Na}]^{+}\right)$.

\section{2,6-Dichloro- $N, N$-dimethylaniline (5)}

To a solution of 2,6-dichloroaniline $(1.0 \mathrm{~g}, 6.2 \mathrm{mmol})$ in toluene $(10 \mathrm{~mL})$ dimethyl sulfate $(3.13 \mathrm{~g}, 24.5 \mathrm{mmol})$ and anhydrous potassium carbonate $(858 \mathrm{mg}, 6.2 \mathrm{mmol})$ were added, and the mixture was refluxed overnight. After cooling to r.t. aq. ammonia solution $(25 \%, 20 \mathrm{~mL})$ was added, and the mixture was stirred for $5 \mathrm{~min}$. Then ethyl acetate $(20 \mathrm{~mL})$ was added, the fractions were separated, and the organic fraction was washed twice with water. The product was purified by column chromatography (petroleum etherethyl acetate $10: 1)$, then isolated as a colorless liquid; yield: $300 \mathrm{mg}(25 \%) .-{ }^{1} \mathrm{H}$ NMR (400 MHz, $\mathrm{CDCl}_{3}$ ): $\delta=2.89$ (s, $6 \mathrm{H}, M e), 6.96(\mathrm{t}, J=7.8 \mathrm{~Hz}, 1 \mathrm{H}, H-4), 7.25(\mathrm{~d}, J=7.8 \mathrm{~Hz}$, $2 \mathrm{H}, H-3, H-5) .-{ }^{13} \mathrm{C} \mathrm{NMR}\left(100 \mathrm{MHz}, \mathrm{CDCl}_{3}\right): \delta=42.1$ (2 C, Me), 125.8 (1 C, C-4), 129.1 (2 C, $C-3, C-5), 135.5$ (2 C, $C$-2, $C$-6), 146.4 (1 C, $C$-1). The NMR data were in accordance with the literature [8].

\section{2,4,6-Trichloro- $N, N$-dimethylaniline (6)}

2,4,6-Trichloro- $N, N$-dimethylaniline (6) was prepared according to the procedure for $\mathbf{5}$ from 2,4,6-trichloroaniline $(1.0 \mathrm{~g}, 5.1 \mathrm{mmol})$, toluene $(8 \mathrm{~mL})$, dimethyl sulfate $(2.59 \mathrm{~g}$, $20.5 \mathrm{mmol}$ ), and anhydrous potassium carbonate $(709 \mathrm{mg}$, $5.1 \mathrm{mmol})$. The product was isolated as a colorless liquid; yield: $579 \mathrm{mg}(51 \%) .-{ }^{1} \mathrm{H}$ NMR $\left(400 \mathrm{MHz}, \mathrm{CDCl}_{3}\right): \delta=$ $2.86(\mathrm{~s}, 6 \mathrm{H}, M e), 7.27(\mathrm{~s}, 2 \mathrm{H}, H-3, H-5) .-{ }^{13} \mathrm{C} \mathrm{NMR}$ $\left(100 \mathrm{MHz}, \mathrm{CDCl}_{3}\right): \delta=42.0(2 \mathrm{C}, \mathrm{Me}), 128.8(2 \mathrm{C}, \mathrm{C}-3, C$ 5), 130.1 (1 C, $C$-4), 135.9 (2 C, $C-2, C-6), 145.3$ (1 C, $C-1$ ). The NMR data were in accordance with the literature [8].

\section{3-Chloro-1-methyl-1H-indole (8)}

3-Chloro-1-methyl-1H-indole (8) was synthesized according to the literature for the synthesis of 3-chloro- $1 \mathrm{H}$ - 
indole [9]. Under an atmosphere of nitrogen $N$-chlorosuccinimide (214 mg, $1.53 \mathrm{mmol}$ ) was added to a solution of 1methyl- $1 H$-indole (7) $(200 \mathrm{mg}, 1.53 \mathrm{mmol})$ in anhydrous DMF $(6 \mathrm{~mL})$. The reaction mixture was stirred for $2 \mathrm{~h}$ at $\mathrm{r}$. t. in the dark, and then brine $(10 \mathrm{~mL})$ was added. The aq. fraction was extracted with ethyl acetate $(3 \times 30 \mathrm{~mL})$, and the organic fraction was washed with water and dried (magnesium sulfate). After evaporation of the solvent the product was purified by column chromatography (petroleum ether-ethyl acetate $8: 1$ ). The product was isolated as a light-yellow oil; yield: $147 \mathrm{mg}(58 \%) .-{ }^{1} \mathrm{H} \mathrm{NMR}\left(400 \mathrm{MHz}, \mathrm{CDCl}_{3}\right): \delta=$ $3.74(\mathrm{~s}, 3 \mathrm{H}, \mathrm{Me}), 7.01(\mathrm{~s}, 1 \mathrm{H}, \mathrm{NCH}), 7.16-7.20(\mathrm{~m}, 1 \mathrm{H}$, $H-6), 7.24-7.31(\mathrm{~m}, 2 \mathrm{H}, H-5, H-7), 7.61-7.63(\mathrm{~m}, 1 \mathrm{H}$, $H-4) .-{ }^{13} \mathrm{C} \mathrm{NMR}\left(100 \mathrm{MHz}, \mathrm{CDCl}_{3}\right): \delta=32.9(1 \mathrm{C}, \mathrm{Me})$, 104.4 (1 C, CCl), 109.5 (1 C), 118.3 (1 C), 119.9 (1 C), 122.6 (1 C), 125.2 (1 C), 125.7 (1 C), 135.8 (1 C). - MS (EI, 70 eV): $m / z(\%)=165(100)[\mathrm{M}]^{+}, 150(13), 128(13), 101(16)$. The NMR data were in accordance with the literature [10].

\section{3-Chloro-1-methyl-1H-indole-2-carbaldehyde (9) / 1-meth- yl-1H-indole-3-carbaldehyde (10)}

The product was synthesized according to the general procedure from 3-chloro-1-methyl-1H-indole $(\mathbf{8})(100 \mathrm{mg}$, $0.606 \mathrm{mmol})$, anhydrous DMF $(1 \mathrm{~mL}), 1.1$ eq. of $\mathrm{POCl}_{3}$ $(0.061 \mathrm{~mL}, 0.666 \mathrm{mmol}) ; 50{ }^{\circ} \mathrm{C}, 3 \mathrm{~h}$, ice water $(5 \mathrm{~mL})$, sat. aq. solution of sodium hydrogen carbonate $(10 \mathrm{~mL}), \mathrm{CH}_{2} \mathrm{Cl}_{2}$ $(3 \times 10 \mathrm{~mL})$; column chromatography (petroleum etherethyl acetate $2: 1$ ). The products were isolated as colorless solids.

3-Chloro-1-methyl-1H-indole-2-carbaldehyde (9): yield: $35 \mathrm{mg}(30 \%)$, m.p. $86{ }^{\circ} \mathrm{C} .-{ }^{1} \mathrm{H}$ NMR (400 MHz, $\left.\mathrm{CDCl}_{3}\right)$ : $\delta=3.75(\mathrm{~s}, 3 \mathrm{H}, \mathrm{Me}), 7.26-7.32\left(\mathrm{~m}, 3 \mathrm{H}, H_{\text {arom }}\right), 8.25-8.27$ $\left(\mathrm{m}, 1 \mathrm{H}, H_{\text {arom }}\right), 10.07(\mathrm{~s}, 1 \mathrm{H}, \mathrm{CHO}) .-{ }^{13} \mathrm{C} \mathrm{NMR}(100 \mathrm{MHz}$, $\left.\mathrm{CDCl}_{3}\right): \delta=30.1(1 \mathrm{C}, \mathrm{Me}), 109.4(1 \mathrm{C}), 112.8(1 \mathrm{C}), 121.2$ (1 C), 123.5 (1 C), 124.0 (1 C), 124.2 (1 C), 135.9 (1 C), 136.7 (1 C), 183.8 (1 C, CHO). - MS (EI, $70 \mathrm{eV}): \mathrm{m} / z(\%)=$ $192(100)[\mathrm{M}-\mathrm{H}]^{+}, 128(17), 101$ (18). The NMR data were in accordance with the literature [10].

1-Methyl-1H-indole-3-carbaldehyde (10): yield: $3 \mathrm{mg}$ (3\%), m. p. $70{ }^{\circ} \mathrm{C} .-{ }^{1} \mathrm{H}$ NMR $\left(400 \mathrm{MHz}, \mathrm{CDCl}_{3}\right): \delta=3.88$ (s, $3 \mathrm{H}, M e), 7.32-7.38$ (m, $\left.3 \mathrm{H}, H_{\text {arom }}\right), 7.69$ (s, $\left.1 \mathrm{H}, H-2\right)$, 8.29-8.32 (m, $\left.1 \mathrm{H}, H_{\text {arom }}\right), 10.00$ (s, $\left.1 \mathrm{H}, \mathrm{CHO}\right)$. The NMR data were in accordance with the literature [10].

\section{5-Halo-2-thiophenecarbaldehydes 14-16}

The products were synthesized according to the general procedure from 2-halothiophene $(3.0 \mathrm{mmol})$, anhydrous DMF (6 mL), and 2.2 eq. of $\mathrm{POCl}_{3}(0.6 \mathrm{~mL}, 6.6 \mathrm{mmol})$; $100{ }^{\circ} \mathrm{C}, 4 \mathrm{~h}$, ice water $(5 \mathrm{~mL})$, sat. aq. solution of sodium hydrogen carbonate $(10 \mathrm{~mL}), \mathrm{CH}_{2} \mathrm{Cl}_{2}(3 \times 10 \mathrm{~mL})$; column chromatography (pentane- $\mathrm{CH}_{2} \mathrm{Cl}_{2} 1: 1$ ).
Starting material: 2-chlorothiophene (11); product: 5chlorothiophene-2-carbaldehyde (14). The product was isolated as a colorless liquid; yield: $35 \mathrm{mg}(8 \%)$.

Starting material: 2-bromothiophene (12); product: 5chlorothiophene-2-carbaldehyde (14). The product was isolated as a colorless liquid; yield: $37 \mathrm{mg} \mathrm{(8 \% ).}$

Starting material: 2-iodothiophene (13); products: mixture of 5-chloro-thiophene-2-carbaldehyde (14) / 5-iodothiophene-2-carbaldehyde (16) $1: 0.33$. The products were isolated as a colorless liquid; yield (both products): $99 \mathrm{mg}$ $(20 \%)$.

5-Chlorothiophene-2-carbaldehyde (14): ${ }^{1} \mathrm{H}$ NMR (400 $\mathrm{MHz}, \mathrm{CDCl}_{3}$ ): $\delta=7.05$ (d, $\left.J=4.0 \mathrm{~Hz}, 1 \mathrm{H}, H_{\text {arom }}\right), 7.57$ (d, $\left.J=4.0 \mathrm{~Hz}, 1 \mathrm{H}, H_{\text {arom }}\right), 9.78$ (s, $\left.1 \mathrm{H}, \mathrm{CHO}\right)$. The NMR data were in accordance with the literature [10].

5-Iodothiophene-2-carbaldehyde (16): ${ }^{1} \mathrm{H}$ NMR (400 $\mathrm{MHz}, \mathrm{CDCl}_{3}$ ): $\delta=7.39$ (s, $\left.2 \mathrm{H}, H_{\text {arom }}\right), 9.77$ (s, $1 \mathrm{H}, \mathrm{CHO}$ ). The NMR data were in accordance with the literature [14].

\section{4-(Thien-3-yl)morpholine (20)}

4-(Thien-3-yl)morpholine (20) was synthesized according to the literature [15] from 3-bromothiophene. The product was isolated as a colorless solid; yield: $499 \mathrm{mg}(80 \%)$, m.p. $90{ }^{\circ} \mathrm{C} .-{ }^{1} \mathrm{H}$ NMR $\left(400 \mathrm{MHz}, \mathrm{CDCl}_{3}\right): \delta=3.07-3.10$ (m, $\left.4 \mathrm{H}, \mathrm{CH}_{2} \mathrm{NCH}_{2}\right), 3.83-3.85\left(\mathrm{~m}, 4 \mathrm{H}, \mathrm{CH}_{2} \mathrm{OCH}_{2}\right), 6.20$ (dd, $\left.J=3.0,1.6 \mathrm{~Hz}, 1 \mathrm{H}, H-2_{\text {arom }}\right), 6.86(\mathrm{dd}, J=5.2,1.6 \mathrm{~Hz}$, $\left.1 \mathrm{H}, H-4_{\text {arom }}\right), 7.25$ (dd, $\left.J=5.2,3.0 \mathrm{~Hz}, 1 \mathrm{H}, H-5_{\text {arom }}\right)$. ${ }^{13} \mathrm{C}$ NMR (100 MHz, $\left.\mathrm{CDCl}_{3}\right): \delta=50.7\left(2 \mathrm{C}, \mathrm{CH}_{2} \mathrm{NCH}_{2}\right)$, $66.6\left(2 \mathrm{C}, \mathrm{CH}_{2} \mathrm{OCH}_{2}\right), 100.4\left(1 \mathrm{C}, C-2_{\text {arom }}\right), 119.6$ (1 C, $C$ $\left.4_{\text {arom }}\right), 125.5$ (2 C, $C$-5 arom $), 152.4$ (2 C, $C$-3 $3_{\text {arom }}$ ). - MS (EI, $70 \mathrm{eV}): m / z(\%)=169(54)[\mathrm{M}]^{+}, 149(21), 125(13), 111$ (100), 97 (32). The NMR data were in accordance with the literature [15].

\section{3-(4-Morpholino)thiophene-2-carbaldehyde (21)}

Method I: The product was synthesized according to the general procedure from 4-(thienyl-3-yl)morpholine (20) (75 mg, $0.444 \mathrm{mmol})$, anhydrous DMF $(0.6 \mathrm{~mL})$, and 1.1 eq. of $\mathrm{POCl}_{3}(0.037 \mathrm{~mL}, 0.488 \mathrm{mmol}) ; 50{ }^{\circ} \mathrm{C} ; 1.5 \mathrm{~h}$, ice water $(5 \mathrm{~mL})$, sat. aq. solution of sodium hydrogen carbonate $(10 \mathrm{~mL}), \mathrm{CH}_{2} \mathrm{Cl}_{2}(3 \times 10 \mathrm{~mL})$; column chromatography (petroleum ether-ethyl acetate $1: 1$ ). The product was isolated as a yellow solid; yield: $54 \mathrm{mg}(62 \%)$, m. p. $55^{\circ} \mathrm{C}$.

Method II: The product was synthesized according to the general procedure from 4-(2-bromo-3-thienyl)morpholine (23) (20 mg, $0.081 \mathrm{mmol})$, anhydrous DMF (0.12 mL), and 1.1 eq. of $\mathrm{POCl}_{3}(0.008 \mathrm{~mL}, 0.089 \mathrm{mmol}) ; 50{ }^{\circ} \mathrm{C}, 4.5 \mathrm{~h}$, ice water $(5 \mathrm{~mL})$, sat. aq. solution of sodium hydrogen carbonate $(5 \mathrm{~mL}), \mathrm{CH}_{2} \mathrm{Cl}_{2}(3 \times 5 \mathrm{~mL})$; column chromatography (petroleum ether-ethyl acetate $2: 1$ ). The product was isolated as a yellow solid; yield: $4 \mathrm{mg}(25 \%)$, m.p. $55{ }^{\circ} \mathrm{C}$. ${ }^{1} \mathrm{H}$ NMR $\left(400 \mathrm{MHz}, \mathrm{CDCl}_{3}\right): \delta=3.33-3.36(\mathrm{~m}, 4 \mathrm{H}$, 
$\left.\mathrm{CH}_{2} \mathrm{NCH}_{2}\right), 3.85-3.87\left(\mathrm{~m}, 4 \mathrm{H}, \mathrm{CH}_{2} \mathrm{OCH}_{2}\right), 6.82(\mathrm{~d}, J=$ $\left.5.3 \mathrm{~Hz}, 1 \mathrm{H}, H_{\text {arom }}\right), 7.62\left(\mathrm{dd}, J=5.3,0.8 \mathrm{~Hz}, 1 \mathrm{H}, H_{\text {arom }}\right)$, $9.87(\mathrm{~d}, J=0.8 \mathrm{~Hz}, 1 \mathrm{H}, \mathrm{CHO}) .-{ }^{13} \mathrm{C}$ NMR $(100 \mathrm{MHz}$, $\left.\mathrm{CDCl}_{3}\right): \delta=53.1\left(2 \mathrm{C}, \mathrm{CH}_{2} \mathrm{NCH}_{2}\right), 66.7\left(2 \mathrm{C}, \mathrm{CH}_{2} \mathrm{OCH}_{2}\right)$, 120.9 ( $\left.1 \mathrm{C}, C-4_{\text {arom }}\right), 123.5$ (1 C, $\left.C-2_{\text {arom }}\right), 135.7$ (1 C, $C$ 5 arom $), 157.8$ (1 C, $\left.C-3_{\text {arom }}\right), 180.8$ ( $\left.1 \mathrm{C}, C \mathrm{HO}\right) .-\mathrm{IR}(\mathrm{KBr})$ : $v=3114,2965,2917,2892,2862,2833,1641,1529,1448$, 1428, 1370. 1358, 1310, 1255, 1191, 1168, 1110, 1066, $1028,999,923,884,853,845,819,756,703 \mathrm{~cm}^{-1}$. - MS (EI, $70 \mathrm{eV}): m / z(\%)=197(63)[\mathrm{M}]^{+}, 180(31), 166(100)$, 149 (13), 140 (49), 125 (16), 111 (83), 105 (19), 97 (19), 91 (18). - HRMS ((+)-ESI): $m / z=198.0589$ (calcd. 198.0589 for $\mathrm{C}_{9} \mathrm{H}_{12} \mathrm{NO}_{2} \mathrm{~S},[\mathrm{M}+\mathrm{H}]^{+}$).

\section{4-(2-Chloro-3-thienyl)morpholine (22)}

Under an atmosphere of nitrogen and at $0{ }^{\circ} \mathrm{C} N$-chlorosuccinimide (124 $\mathrm{mg}, 0.931 \mathrm{mmol}$ ) was added in small portions to a solution of 4-(thien-3-yl)morpholine (20) $(150 \mathrm{mg}$, $0.887 \mathrm{mmol})$ in anhydrous DMF $(7 \mathrm{~mL})$. The reaction mixture was stirred for $1 \mathrm{~h}$ at $0{ }^{\circ} \mathrm{C}$ in the dark and then brine $(10 \mathrm{~mL})$ was added. The aq. fraction was extracted with ethyl acetate $(3 \times 15 \mathrm{~mL})$, and the organic fraction was washed with water and dried (magnesium sulfate). After evaporation of the solvent the product was purified by column chromatography (petroleum ether-ethyl acetate $4: 1$ ). The product was isolated as a light-brown oil; yield: $175 \mathrm{mg}(97 \%)$. ${ }^{1} \mathrm{H}$ NMR $\left(400 \mathrm{MHz}, \mathrm{CDCl}_{3}\right): \delta=3.06-3.08(\mathrm{~m}, 4 \mathrm{H}$, $\left.\mathrm{CH}_{2} \mathrm{NCH}_{2}\right), 3.83-3.85\left(\mathrm{~m}, 4 \mathrm{H}, \mathrm{CH}_{2} \mathrm{OCH}_{2}\right), 6.79(\mathrm{~d}, \mathrm{~J}=$ $\left.5.9 \mathrm{~Hz}, 1 \mathrm{H}, H-4_{\text {arom }}\right), 7.04$ (d, $\left.J=5.9 \mathrm{~Hz}, 1 \mathrm{H}, H-5_{\text {arom }}\right)$. ${ }^{13} \mathrm{C} \mathrm{NMR}\left(100 \mathrm{MHz}, \mathrm{CDCl}_{3}\right): \delta=51.5\left(2 \mathrm{C}, \mathrm{CH}_{2} \mathrm{NCH}_{2}\right)$, $67.1\left(2 \mathrm{C}, \mathrm{CH}_{2} \mathrm{OCH}_{2}\right), 114.0(1 \mathrm{C}, \mathrm{CCl}), 120.1(1 \mathrm{C}, C-$ $\left.4_{\text {arom }}\right), 121.6$ (1 C, $C$-5 arom $), 146.9$ ( $\left.1 \mathrm{C}, C-3_{\text {arom }}\right)$ - - MS (EI, $70 \mathrm{eV}): m / z(\%)=203(67)[\mathrm{M}]^{+}, 145(100)$.

\section{4-(2-Bromo-3-thienyl)morpholine (23)}

Under an atmosphere of nitrogen and at $0{ }^{\circ} \mathrm{C} N$-bromosuccinimide (22 $\mathrm{mg}, 0.124 \mathrm{mmol}$ ) was added in small portions to a solution of 4-(thien-3-yl)morpholine (20) (20 mg, $0.118 \mathrm{mmol})$ in anhydrous DMF $(0.9 \mathrm{~mL})$. The reaction mixture was stirred for $1 \mathrm{~h}$ at $0{ }^{\circ} \mathrm{C}$ in the dark, and then brine $(10 \mathrm{~mL})$ was added. The aq. fraction was extracted with ethyl acetate $(3 \times 15 \mathrm{~mL})$, and the organic fraction was washed with water and dried (magnesium sulfate). After evaporation of the solvent the product was purified by column chromatography (petroleum ether-ethyl acetate $4: 1$ ) and isolated as a colorless oil; yield: $22 \mathrm{mg}(75 \%) .-{ }^{1} \mathrm{H}$ NMR (400 MHz, $\left.\mathrm{CDCl}_{3}\right): \delta=3.04-3.07\left(\mathrm{~m}, 4 \mathrm{H}, \mathrm{CH}_{2} \mathrm{NCH}_{2}\right), 3.84-3.86(\mathrm{~m}$, $\left.4 \mathrm{H}, \mathrm{CH}_{2} \mathrm{OCH}_{2}\right), 6.80\left(\mathrm{~d}, J=5.8 \mathrm{~Hz}, 1 \mathrm{H}, H-4_{\text {arom }}\right), 7.22(\mathrm{~d}$, $\left.J=5.8 \mathrm{~Hz}, 1 \mathrm{H}, H-5_{\text {arom }}\right) .-{ }^{13} \mathrm{C} \mathrm{NMR}\left(100 \mathrm{MHz}, \mathrm{CDCl}_{3}\right)$ : $\delta=51.9\left(2 \mathrm{C}, \mathrm{CH}_{2} \mathrm{NCH}_{2}\right), 67.1\left(2 \mathrm{C}, \mathrm{CH}_{2} \mathrm{OCH}_{2}\right), 99.0(1 \mathrm{C}$, $C \mathrm{Br}), 120.5$ (1 C, $C$-4 arom), 125.0 (1 C, $\left.C-5_{\text {arom }}\right), 149.4$ (1 C, $C-3$ arom). - MS (EI, $70 \mathrm{eV}): m / z(\%)=247(53)[\mathrm{M}]^{+}, 189$ (100), 149 (20), 123 (17), 111 (29), 97 (41).

\section{4-(2,5-Dichloro-3-thienyl)morpholine (24)}

This compound was synthesized as described above for 20 [15] from 3-bromo-2,5-dichlorothiophene (1.000 g, $4.351 \mathrm{mmol}$ ) as starting material and with a reaction time of $1.5 \mathrm{~d}$. The product was purified by column chromatography (petroleum ether-ethyl acetate $6: 1$ ) and isolated as a light-brown solid; yield: $74 \mathrm{mg}(7 \%)$, m. p. $65{ }^{\circ} \mathrm{C} .-{ }^{1} \mathrm{H}$ NMR (400 MHz, $\left.\mathrm{CDCl}_{3}\right): \delta=3.01-3.04\left(\mathrm{~m}, 4 \mathrm{H}, \mathrm{CH}_{2} \mathrm{NCH}_{2}\right.$ ), $3.81-3.83\left(\mathrm{~m}, 4 \mathrm{H}, \mathrm{CH}_{2} \mathrm{OCH}_{2}\right), 6.67$ (s, $\left.1 \mathrm{H}, \mathrm{H}-4_{\text {arom }}\right)$. ${ }^{13} \mathrm{C}$ NMR $\left(100 \mathrm{MHz}, \mathrm{CDCl}_{3}\right): \delta=51.4\left(2 \mathrm{C}, \mathrm{CH}_{2} \mathrm{NCH}_{2}\right)$, $66.9\left(2 \mathrm{C}, \mathrm{CH}_{2} \mathrm{OCH}_{2}\right), 110.9$ (1 C, $\left.C-2_{\text {arom }}\right), 119.9$ (1 C, $\left.C-4_{\text {arom }}\right), 125.4$ (1 C, $\left.C-5_{\text {arom }}\right), 146.0$ (1 C, C- 3 arom $)$. - IR $(\mathrm{KBr}): v=2961,2910,2892,2855,2827,1554,1450,1380$, 1353, 1332, 1305, 1281, 1264, 1199, 1170, 1118, 1085, 1069, 1037, 1009, 987, 922, 866, 811, $703 \mathrm{~cm}^{-1}$. - MS (EI, $70 \mathrm{eV}): m / z(\%)=237(48)[\mathrm{M}]^{+}, 202(10), 179(100), 105$ (14).

5-Chloro-3-(4-morpholino)thiophene-2-carbaldehyde (25)

The product was synthesized according to the general procedure from 4-(2,5-dichloro-3-thienyl)morpholine (24) (50 $\mathrm{mg}, 0.211 \mathrm{mmol})$, anhydrous DMF $(0.4 \mathrm{~mL})$, and 1.1 eq. of $\mathrm{POCl}_{3}(0.021 \mathrm{~mL}, 0.232 \mathrm{mmol}) ; 50{ }^{\circ} \mathrm{C}, 1 \mathrm{~h}$, ice water $(5 \mathrm{~mL})$, sat. aq. solution of sodium hydrogen carbonate $(10 \mathrm{~mL}), \mathrm{CH}_{2} \mathrm{Cl}_{2}(3 \times 10 \mathrm{~mL})$; column chromatography (petroleum ether-ethyl acetate $4: 1$ ). The product was isolated as a light-yellow solid; yield: $16 \mathrm{mg}(33 \%)$, m.p. $132{ }^{\circ} \mathrm{C}$. $-{ }^{1} \mathrm{H} \mathrm{NMR}\left(400 \mathrm{MHz}, \mathrm{CDCl}_{3}\right): \delta=3.33-3.36$ (m, $\left.4 \mathrm{H}, \mathrm{CH}_{2} \mathrm{NCH}_{2}\right), 3.83-3.86\left(\mathrm{~m}, 4 \mathrm{H}, \mathrm{CH}_{2} \mathrm{OCH}_{2}\right), 6.69$ (s, $\left.1 \mathrm{H}, H-4_{\text {arom }}\right), 9.76$ (s, $\left.1 \mathrm{H}, \mathrm{CHO}\right) .-{ }^{13} \mathrm{C} \mathrm{NMR}(100 \mathrm{MHz}$, $\left.\mathrm{CDCl}_{3}\right): \delta=52.8\left(2 \mathrm{C}, \mathrm{CH}_{2} \mathrm{NCH}_{2}\right), 66.5\left(2 \mathrm{C}, \mathrm{CH}_{2} \mathrm{OCH}_{2}\right)$, 120.7 (1 C, $\left.C-4_{\text {arom }}\right), 122.0$ ( $\left.1 \mathrm{C}, C-2_{\text {arom }}\right), 142.3$ (1 C, $C$ 5 arom $), 156.3$ ( $\left.1 \mathrm{C}, C-3_{\text {arom }}\right), 179.4$ ( $\left.1 \mathrm{C}, C \mathrm{HO}\right) .-\mathrm{IR}(\mathrm{KBr})$ : $v=3103,2968,2926,2904,2859,1595,1552,1482,1445$, $1426,1374,1356,1329,1308,1284,1256,1208,1161$, 1117, 1091, 1066, 1027, 994, 874, 836, $702 \mathrm{~cm}^{-1}$. - MS (EI, $70 \mathrm{eV}): m / z(\%)=231(75)[\mathrm{M}]^{+}, 214(50), 200(88), 188$ (73), 174 (69), 145 (72), 120 (57), 110 (100), 105 (650), 91 (26).

\section{2-(3,4-Ethylenedioxythiophene) carbaldehyde (27)}

The product was synthesized according to the general procedure from 3,4-ethylenedioxythiophene (26) $(150 \mathrm{mg}$, $1.06 \mathrm{mmol})$, anhydrous DMF (1.5 mL), and 1.1 eq. of $\mathrm{POCl}_{3}$ $(0.11 \mathrm{~mL}, 1.16 \mathrm{mmol}), 50{ }^{\circ} \mathrm{C}, 1.5 \mathrm{~h}$; ice water $(15 \mathrm{~mL})$, sat. aq. solution of sodium hydrogen carbonate $(15 \mathrm{~mL})$, $\mathrm{CH}_{2} \mathrm{Cl}_{2}(3 \times 15 \mathrm{~mL})$; column chromatography (petroleum ether-ethyl acetate $2: 1$ ). The product was isolated as a colorless solid; yield: $148 \mathrm{mg}(83 \%)$, m.p. $141{ }^{\circ} \mathrm{C} .-{ }^{1} \mathrm{H}$ NMR $\left(600 \mathrm{MHz}, \mathrm{CDCl}_{3}\right): \delta=4.26-4.27\left(\mathrm{~m}, 2 \mathrm{H}, \mathrm{OCH}_{2} \mathrm{CH}_{2} \mathrm{O}\right)$, $4.35-4.37\left(\mathrm{~m}, 2 \mathrm{H}, \mathrm{OCH}_{2} \mathrm{CH}_{2} \mathrm{O}\right), 6.79(\mathrm{~d}, J=1.2 \mathrm{~Hz}, 1 \mathrm{H}$, 
$\left.H-5_{\text {arom }}\right), 9.90(\mathrm{~d}, J=1.2 \mathrm{~Hz}, 1 \mathrm{H}, \mathrm{CHO}) .-{ }^{13} \mathrm{C} \mathrm{NMR}$ $\left(150 \mathrm{MHz}, \mathrm{CDCl}_{3}\right): \delta=64.3\left(1 \mathrm{C}, \mathrm{OCH}_{2} \mathrm{CH}_{2} \mathrm{O}\right), 65.3(1 \mathrm{C}$, $\left.\mathrm{OCH}_{2} \mathrm{CH}_{2} \mathrm{O}\right), 110.7$ (1 C, $C-5$ arom $), 118.5$ (1 C4, $\left.C-2_{\text {arom }}\right)$, 141.8 ( $\left.1 \mathrm{C}, C-3_{\text {arom }}\right), 148.4$ ( $1 \mathrm{C}, C-4$ arom $), 180.1$ ( $\left.1 \mathrm{C}, C H O\right)$. The NMR data were in accordance with the literature [17].

\section{2,5-Dibromo-3,4-ethylenedioxythiophene (28)}

The synthesis according to the literature [18] afforded $\mathbf{2 8}$ as a colorless solid; yield: $939 \mathrm{mg}(89 \%)$, m.p. $98{ }^{\circ} \mathrm{C}$. ${ }^{1} \mathrm{H}$ NMR $\left(400 \mathrm{MHz}, \mathrm{CDCl}_{3}\right): \delta=4.27(\mathrm{~s}, 4 \mathrm{H}$, $\left.\mathrm{OCH}_{2} \mathrm{CH}_{2} \mathrm{O}\right) .-{ }^{13} \mathrm{C}$ NMR $\left(100 \mathrm{MHz}, \mathrm{CDCl}_{3}\right): \delta=64.9$ $\left(2 \mathrm{C}, \mathrm{OCH}_{2} \mathrm{CH}_{2} \mathrm{O}\right), 85.5$ (2 C, $\left.\mathrm{CBr}\right), 139.7$ (2 C, OC arom $)$. IR (KBr): $v=2952,2922,2878,1598,1513,1454,1415$, 1362, 1164, 1087, 1039, 977, 931,903, 839, $749 \mathrm{~cm}^{-1}$. MS (EI, $70 \mathrm{eV}): m / z(\%)=300(100)[\mathrm{M}]^{+}, 273(24), 188$ (35), 163 (20), 147 (23), 123 (78), 105 (92), 95 (72), 91 (41).

\section{2-Bromo-3,4-ethylenedioxythiophene (29)}

This compound was synthesized in a process similar to the literature protocol [19]. Under an atmosphere of nitrogen $N$-bromosuccinimide (376 mg, $2.11 \mathrm{mmol}$ ) was added in small portions to a solution of 3,4-ethylenedioxythiophene (26) $(300 \mathrm{mg}, 2.11 \mathrm{mmol})$ in anhydrous DMF (3.5 mL). The reaction mixture was stirred for $1 \mathrm{~h}$ at $\mathrm{r}$.t. in the dark, and was then poured into ice water and extracted with diethyl ether $(3 \times 30 \mathrm{~mL})$. The organic fraction was washed with brine and dried (magnesium sulfate). After evaporation of the solvent the product was purified by column chromatography (pentane-THF 20:1). The product was isolated as a colorless oil; yield: $243 \mathrm{mg}(52 \%) .-{ }^{1} \mathrm{H}$ NMR $(400 \mathrm{MHz}$, $\left.\mathrm{CDCl}_{3}\right): \delta=4.18-4.21\left(\mathrm{~m}, 2 \mathrm{H}, \mathrm{OCH}_{2} \mathrm{CH}_{2} \mathrm{O}\right), 4.25-4.28$ $\left(\mathrm{m}, 2 \mathrm{H}, \mathrm{OCH}_{2} \mathrm{CH}_{2} \mathrm{O}\right), 6.34$ (s, $\left.1 \mathrm{H}, \mathrm{H}-5_{\text {arom }}\right) .-{ }^{13} \mathrm{C} \mathrm{NMR}$ $\left(100 \mathrm{MHz}, \mathrm{CDCl}_{3}\right): \delta=64.5\left(1 \mathrm{C}, \mathrm{OCH}_{2} \mathrm{CH}_{2} \mathrm{O}\right), 65.0$
(1 C, $\left.\mathrm{OCH}_{2} \mathrm{CH}_{2} \mathrm{O}\right), 87.0$ (1 C, $\left.C \mathrm{Br}\right), 99.6$ (1 C, $C-5_{\text {arom }}$ ), 140.0 ( $1 \mathrm{C}, C-3_{\text {arom }}$ or $\left.C-4_{\text {arom }}\right), 141.1$ (1 C, $C-3_{\text {arom or }}$ $\left.C-4_{\text {arom }}\right)$. - IR (KBr): $v=3112,2984,2927,2874,1497$, 1450, 1418, 1363, 1269, 1246, 1183, 1154, 1070, 1036, 926, $899,712 \mathrm{~cm}^{-1}$. - MS (EI, $\left.70 \mathrm{eV}\right): \mathrm{m} / z(\%)=220(100)$ $[\mathrm{M}]^{+}, 194$ (13), 141 (28), 120 (31), 105 (73), 97 (27), 91 (28).

5-Bromo-3,4-ethylenedioxythiophene-2-carbaldehyde (30) / 5-chloro-3,4-ethylenedioxythiophene-2-carbaldehyde (31)

Method I: The products were synthesized according to the general procedure from 2-bromo-3,4-ethylenedioxythiophene (29) (100 mg, $0.452 \mathrm{mmol})$, anhydrous DMF $(0.7 \mathrm{~mL})$, and 1.1 eq. of $\mathrm{POCl}_{3}(0.045 \mathrm{~mL}, 0.497 \mathrm{mmol})$; $50{ }^{\circ} \mathrm{C}, 3 \mathrm{~h}$, ice water $(15 \mathrm{~mL})$, sat. aq. solution of sodium hydrogen carbonate $(15 \mathrm{~mL}), \mathrm{CH}_{2} \mathrm{Cl}_{2}(3 \times 15 \mathrm{~mL})$; column chromatography (petroleum ether-ethyl acetate $2: 1$ ). The products were isolated as colorless solids, a 1.00:0.22 mixture of $\mathbf{3 0}$ and 31; yield (both products): $91 \mathrm{mg}$ (84\%).

Method II: The products were synthesized according to the general procedure from 2,5-dibromo-3,4-ethylenedioxythiophene (28) (100 mg, $0.333 \mathrm{mmol})$, anhydrous DMF $(0.5 \mathrm{~mL})$, and 1.1 eq. of $\mathrm{POCl}_{3}(0.033 \mathrm{~mL}, 0.366 \mathrm{mmol})$; $50{ }^{\circ} \mathrm{C}, 1 \mathrm{~d}$, ice water $(15 \mathrm{~mL})$, sat. aq. solution of sodium hydrogen carbonate $(15 \mathrm{~mL}), \mathrm{CH}_{2} \mathrm{Cl}_{2}(3 \times 15 \mathrm{~mL})$; column chromatography (petroleum ether-ethyl acetate $2: 1$ ). The products were isolated as colorless solids, a $0.32: 1.00 \mathrm{mix}$ ture of 30 and 31; yield (both products): $47 \mathrm{mg}$ (66\%).

5-Bromo-3,4-ethylenedioxythiophene-2-carbaldehyde (30): ${ }^{1} \mathrm{H} \mathrm{NMR}\left(400 \mathrm{MHz}, \mathrm{CDCl}_{3}\right): \delta=4.34-4.39(\mathrm{~m}, 4 \mathrm{H}$, $\left.\mathrm{OCH}_{2} \mathrm{CH}_{2} \mathrm{O}\right), 9.85$ (s, $\left.1 \mathrm{H}, \mathrm{CHO}\right)$.

5-Chloro-3,4-ethylenedioxythiophene-2-carbaldehyde (31): ${ }^{1} \mathrm{H}$ NMR (400 MHz, $\left.\mathrm{CDCl}_{3}\right): \delta=4.34-4.39(\mathrm{~m}, 4 \mathrm{H}$, $\left.\mathrm{OCH}_{2} \mathrm{CH}_{2} \mathrm{O}\right), 9.82(\mathrm{~s}, 1 \mathrm{H}, \mathrm{CHO})$.
[1] a) V. A. Zapol'skii, J.C. Namyslo, M. Gjikaj, D.E. Kaufmann, Z. Naturforsch. 2010, 65b, 843-860; b) V. A. Zapol'skii, R. Fischer, J. C. Namyslo, D. E. Kaufmann, Bioorg. Med. Chem. 2009, 17, 4206-4215; c) E. Nutz, V. A. Zapol'skii, D. E. Kaufmann, Synthesis 2009, 16, 2719-2724; d) V. A. Zapol'skii, J. C. Namyslo, A. E. W. Adam, D. E. Kaufmann, Heterocycles 2004, 63, $1281-1298$.

[2] I. F. Perepichka, D. F. Perepichka (Eds.) Handbook of Thiophene-based Materials, Vol. 1+2, John Wiley, Chichester, 2009.

[3] a) A. Vilsmeier, A. Haack, Ber. Dtsch. Chem. Ges. 1927, 60, 119-122; b) G. Jones, S. P. Stanforthe, Org. React., Vol. 49, John Wiley, New York, 1997, pp. 1 330; c) O. G. Altinbas, D.E. Kaufmann, T. Clark, J. Mol. Model. 2011, 12, 3209-3217.
[4] S. A. Koz'min, N. A. Bumagin, I. P. Beletskaya, Izvest. Akad. Nauk, Ser. Khim. 1993, 3, 615.

[5] H. M. Chawla, N. Pant, B. Srivastava, Tetrahedron Lett. 2005, 46, 7259-7262.

[6] R. V. Kaberdin, V. I. Potkin, V. A. Zapol'skii, Russ. Chem. Rev. 1997, 66, $827-842$.

[7] DFT calculations on the mechanism are in progress.

[8] R. G. Pews, J. E. Hunter, R. M. Wehmeyer, Tetrahedron 1993, 49, 4809- 4820 .

[9] C. A. Kuttruff, H. Zipse, D. Trauner, Angew. Chem. 2011, 123, 1438 -1441; Angew. Chem. Int. Ed. 2011, 50, $1402-1405$.

[10] B. Liégault, I. Petrov, S. I. Gorelsky, K. Fagnou, J. Org. Chem. 2010, 75, 1047-1060.

[11] E. Campaigne, W. L. Archer, J. Am. Chem. Soc. 1953, 75, 989-991. 
[12] P. Rajender Kumar, S. Raju, P. Satish Goud, M. Sailaja, M. R. Sarma, G. Om Reddy, M. Prem Kumar, V. V. R. M. Krishna Reddy, T. Suresha, P. Hegde, Bioorg. Med. Chem. 2004, 12, $1221-1230$.

[13] Y. Liu, N. Xiang, X. Feng, P. Shen, W. Zhou, C. Weng, B. Zhao, S. Tan, Chem. Commun. 2009, 2499-2501.

[14] M. D‘Auria, D. Tofani, Tetrahedron 1992, 48, 9315 9322.

[15] M. D. Charles, P. Schultz, S. L. Buchwald, Org. Lett. 2005, 7, 3965-3968.
[16] S. Kirchmeyer, K. Reuter, J. Mater. Chem. 2005, 15, $2077-2088$.

[17] M. Jessing, M. Brandt, K. J. Jensen, J. B. Christensen, U. Boas, J. Org. Chem. 2006, 71, 6734-6741.

[18] R. G. Hicks, M. B. Nodwell, J. Am. Chem. Soc. 2000, 122, 6746-6753.

[19] M. Belletête, S. Beaupré, J. Bouchard, P. Blondin, M. Leclerc, G. Durocher, J. Phys. Chem. B 2000, 104, 9118-9125. 